Journal of Applied Mathematics and Stochastic Analysis, 16:4 (2003), 311-326.

Printed in the USA (C)2003 by North Atlantic Science Publishing Company

\title{
ON THE ERGODIC DISTRIBUTION OF OSCILLATING QUEUEING SYSTEMS
}

\author{
MYKOLA BRATIYCHUK \\ Silesian University of Technology, Institute of Mathematics \\ Kaszubska 23, 44-100, Gliwice, Poland \\ e-mail: bratiich@polsl.gliwice.pl \\ ANDRZEJ CHYDZINSKI \\ Silesian University of Technology, Institute of Computer Sciences \\ Akademicka 16, 44-100 Gliwice, Poland \\ e-mail: andych@polsl.gliwice.pl
}

(Received April, 2002; Revised March, 2003)

\begin{abstract}
This paper examines a new class of queueing systems and proves a theorem on the existence of the ergodic distribution of the number of customers in such a system. An ergodic distribution is computed explicitly for the special case of a G/M-M/1 system, where the interarrival distribution does not change and both service distributions are exponential. A numerical example is also given.

Keywords: Queueing System, Ergodic Distribution, Potential Method.
\end{abstract}

AMS (MOS) subject classification: 60K25, 68M20, 90B22, 60J45.

\section{Introduction}

In application of queueing systems, the following two problems are of great importance:

a) We don't want the queue to be too long;

b) It is desirable that the total idle time of the server should be as short as possible.

These requirements are in opposition to each other. This situation is readily identified in the following example. Let us consider a standard $G / G / 1$-type queueing system and let $\rho$ be its traffic intensity. It is well known that if $0<\rho<1$, then the mean value of the queue length is finite, but it increases towards infinity as $\rho \uparrow 1$. On the other hand, the mean value of total idle time has a tendency to increase as $\rho \downarrow 0$. So, problem a) requires $\rho$ to be close to 0 , while problem b) requires $\rho$ to be close to 1 . This article proposes to satisfy both these requirements using a special type of queuing system.

One of the most commonly used methods of attacking the above-mentioned problems is to consider systems with variable service intensities, i.e., those whose service rates depend on queue length (see [4] for survey of the subject). But, besides being difficult for practical application, such systems are rather complicated for mathematical treatment. 
Bearing this in mind, we consider the idea of the oscillating random walk (or oscillating random process) which goes back to J. Kemperman's work [6]. After that paper was published, a series of works appeared in which oscillating random processes (both discrete and continuous time versions) were studied in different ways. We exploit this idea as well as Lotov's work [9], that deals with the problem of the existence of the ergodic distribution of the oscillating process, which is closest to our approach. Nevertheless, the specific nature of our process (since we deal with a service process) has forced us to develop quite a different technique.

An important property of the proposed oscillating system is that it is a generalization of many schemes of traffic control in ATM networks studied recently. In these schemes, the idea of a threshold value for the length of a queue is used for reducing/increasing service or arrival rate (see $[3,11,5,8,12])$. For instance, in [3] authors deal with the following system: Customers arrive at the queue by a Poisson process, and there is only one server. If the queue length at service initiation of a customer is less than threshold $L$ (respectively, greater than or equal to threshold $L$ ), the service time of the customer follows a distribution $F_{1}$ (respectively, $F_{2}$ ). Our situation results when specialized to $a=L-1, b=L+1$ and $G_{1}(x)=G_{2}(x)=1-e^{-\lambda x}$ are directly applicable to this model.

\section{Oscillating Queueing System}

The oscillating queueing system (OQS) works like a classical $G I / G I / 1 / \infty$-type system with service discipline FCFS, with one exception: the interarrival and service times of individual customers are not identically distributed, but alternated at particular (random) moments. The following explains the mechanism of these changes.

Let distribution functions $G_{i}(x), F_{i}(x), x>0, i=1,2$ be given and $a, b$ be two fixed natural numbers such that $0<a<b, b-a \geq 2$. Let $\xi(t)$ denote the number of customers in the OQS at moment $t$, including customer in service, if any. It is assumed that the process $\xi(t)$ is right-hand continuous.

Let $\xi(0) \in(0, b)$. Starting from moment $t=0$, the interarrival intervals have distribution $G_{1}(x)$ and the service times are distributed according to $F_{1}(x)$.

Let $\tau^{+}(b, 1)=\inf \{t>0: \xi(t)=b\}$ and $\beta\left(\tau^{+}(b, 1)\right)$ be the remaining service time at moment $\tau^{+}(b, 1)$. Beginning from moment $\tau^{+}(b, 1)$, customers begin to arrive according to the distribution $G_{2}(x)$ i.e., starting from that moment the interarrival intervals have distribution $G_{2}(x)$. As for the server, it must complete the service of the customer that is in service at moment $\tau^{+}(b, 1)$ and only from moment $\tau^{+}(b, 1)+\beta\left(\tau^{+}(b, 1)\right)$ do the service times of individual customers take the new distribution $F_{2}(x)$.

Let $\tau^{-}(a, 1)=\inf \left\{t>\tau^{+}(b, 1): \xi(t)=a\right\}$ and $\beta\left(\tau^{-}(a, 1)\right)$ be the remaining arrival time at the moment $\tau^{-}(a, 1)$. Beginning from moment $\tau^{-}(a, 1)$, the server begins to serve individual customers according to distribution $F_{1}(x)$, but beginning only from moment $\tau^{-}(a, 1)+\beta\left(\tau^{-}(a, 1)\right)$ the interarrival intervals will be distributed according to distribution $G_{1}(x)$.

At moment $\tau^{+}(b, 2)=\inf \left\{t>\tau^{-}(a, 1): \xi(t)=b\right\}$ we change the distributions $G_{1}(x), F_{1}(x)$ to $G_{2}(x), F_{2}(x)$ in the same way as described previously and so on.

If $\xi(0) \geq b$ the changes in our description are obvious.

We denote the above-described system symbolically by $G-G / G-G / 1$.

If $G_{1}(x)=G_{2}(x)$ then, in fact, we have a system with switching service process, 
denoted by $G / G-G / 1$. On the other hand, if $F_{1}(x)=F_{2}(x)$ we can say that we have a system with switching arrival process, denoted by $G-G / G / 1$.

In conclusion, we state the main result of this section. Let

$$
m_{i}(k)=\int_{0}^{\infty} x^{k} d F_{i}(x), \quad M_{i}(k)=\int_{0}^{\infty} x^{k} d G_{i}(x),
$$

and suppose that the following conditions hold:
A: $m_{2}(1)<M_{2}(1)$
B: $m_{2}(2)<\infty, M_{2}(2)<\infty$;
C: $\int_{0}^{\infty}\left(1-G_{1}(h+x)\right) d F_{1}(x)>0$ for some $h>0$;
D: at least one of the distributions $F_{i}(x), G_{i}(x)$ is non-lattice.

Theorem 2.1: If conditions $\mathbf{A}$ - $\mathbf{D}$ hold, then for any $k \geq 0$ there exists

$$
\lim _{t \rightarrow \infty} \mathbf{P}\{\xi(t)=l / \xi(0)=k\}=P_{l} \geq 0, \quad l \geq 0, \quad \sum_{l=0}^{\infty} P_{l}=1 .
$$

Let us make some comments about the conditions A-D.

The standard $G I / G I / 1 / \infty$-type system with interarrival time distribution $G(x)$, service time distribution $F(x)$ and service discipline FCFS will be denoted symbolically as $G I / G I / 1 /(G, F)$

Condition $\mathbf{A}$ is necessary for the existence of ergodic distribution for the length of the queue. Indeed, beginning from moment $\tau^{+}(b, 1)+\beta\left(\tau^{+}(b, 1)\right)$ we have, in fact, a system of the type $G I / G I / 1\left(G_{2}, F_{2}\right)$, and if $m_{2}(1) \geq M_{2}(1)$, such a system does not possess an ergodic distribution.

Condition $\mathbf{C}$ means that if we consider the system $G I / G I / 1 /\left(G_{1}, F_{1}\right)$ with $\tau$ being its residual busy period, then $\mathbf{P}\{\tau<\infty / \xi(0)=n\}>0$ for any $n \geq 1$. This fact we shall use in Lemma 3 below.

Condition B is connected with the method of the proof of the theorem above.

Condition $\mathbf{D}$ serves to simplify some technical details of the proof.

\section{Proof of the Theorem}

The main difficulty in proving Theorem 2 consists in the fact that, generally speaking, process $\xi(t)$ has no Markovian moments with finite mean values. This difficulty will be remedied by using some facts of semi-Markov renewal theory.

Let us define the following events:

$A(n, x), n \geq 1, x>0$ - at moment $t=0$ exactly $n$ customers are in the system. This moment is the departure epoch and the first customer arrives in time $x$.

$D(n, x), n \geq 1, x>0$ - at moment $t=0$ exactly $n$ customers are in the system. This moment is the arrival epoch and the first departure occurs in time $x$.

We put

$$
Q(x, d u, d y)=\mathbf{P}\left\{\tau^{-}(a, 1) \in d u, \beta\left(\tau^{-}(a, 1)\right) \in d y / A(a, x)\right\}
$$


where, we recall, $\tau^{+}(b, 1)=\inf \{t>0: \xi(t)=b\}, \tau^{-}(a, 1)=\inf \left\{t>\tau^{+}(b, 1): \xi(t)=a\right\}$ and $\beta\left(\tau^{-}(a, 1)\right)$ is the remaining arrival time at moment $\tau^{-}(a, 1)$.

It is understood that $Q(x, d u, d y)$ can be interpreted as a transition kernel of some semi-Markov process, but this fact is not considered here.

By the standard arguments we get

$$
\mathbf{P}\{\xi(t)=l / A(a, x)\}=\int_{0}^{t} \int_{0}^{\infty} U_{l}(t-u, y) H(x, d u, d y),
$$

where

$$
\begin{aligned}
H(x, d u, d y) & =\sum_{n=0}^{\infty} Q^{n *}(x, d u, d y) \\
Q^{(n+1) *}(x, d u, d y) & =\int_{0}^{u} \int_{0}^{\infty} Q(x, d v, d z) Q^{n *}(z, d u-v, d y) \\
U_{l}(t, y) & =\mathbf{P}\left\{\xi(t)=l, \tau^{-}(a, 1)>t / A(a, y)\right\} .
\end{aligned}
$$

If we prove that

$$
\lim _{t \rightarrow \infty} \mathbf{P}\{\xi(t)=l / A(a, x)\}=P_{l},
$$

we can complete the proof in an obvious way.

The following theorem proved by Shurenkov [10] plays the central role in proving (3.2), and we state that theorem in a convenient form to be used for our purposes.

Theorem 3.1: Let the kernel $Q(x, d u, d y)$ be non-lattice and the kernel $Q(x, \cdot)=\mathbf{P}\left\{\beta\left(\tau^{-}(a, 1)\right) \in \cdot / A(a, x)\right\}$ satisfies the following conditions.

1) there exists a stationary probabilistic measure $\pi_{+}\{\cdot\}$ for this kernel,

2) $m=\int_{0}^{\infty} \pi_{+}\{d x\} \int_{0}^{\infty} t Q(x, d t)<\infty$.

Then for any non-negative function $g(t, y), t \geq 0, y \geq 0$ which is directly Riemann integrable in $t$ for any $y$ such that

$$
\int_{0}^{\infty} \pi_{+}\{d y\} \int_{0}^{\infty} g(t, y) d t<\infty
$$

we have

$$
\lim _{t \rightarrow \infty} \int_{0}^{t} \int_{0}^{\infty} g(t-u, y) Q(x, d u, d y)=m^{-1} \int_{0}^{\infty} \pi_{+}\{d y\} \int_{0}^{\infty} g(t, y) d t
$$

The non-latticeness of kernel $Q(x, d u, d y)$ is obvious, due to condition $\mathbf{D}$.

Item 1) follows from the next lemma which is, in fact, Deblin's condition for the kernel $Q(x, d u)$. 
Lemma 3.1: There exists a probabilistic measure $\varphi\{\cdot\}$ and some number $0<\varepsilon<1$ such that if $\varphi\{B\}) \leq \varepsilon$ for a Borel set $B \subset[0, \infty)$, then $Q(x, B) \leq 1-\varepsilon$ for all $x \geq 0$.

Proof: Define the measure $\varphi\{B\}$ by

$$
\varphi\{B\}=\mathbf{P}\left\{\beta\left(\tau^{-}(a, 1) \in B / \xi(0)=1\right\} .\right.
$$

Obviously, in our case $\varphi\{[0, \infty)\}=1$. Set

$$
\widehat{\tau}=\inf \left\{t>0: \xi(t)=0, \sup _{u \leq \widehat{\tau}} \xi(u)<b\right\} .
$$

From condition $\mathbf{C}$ it follows that

$$
\delta=\inf _{x \geq 0} \mathbf{P}\{\hat{\tau}<\infty / A(a, x)\}>0 .
$$

Let a Borel set $B \subset[0, \infty)$ be such that $\varphi\{B\}) \leq \delta /(1+\delta)$. We have

$$
\begin{aligned}
Q(x, B) & =1-\mathbf{P}\left\{\beta\left(\tau^{-}(a, 1)\right) \notin B / A(a, x)\right\} \\
& \leq 1-\int_{0}^{\infty} \mathbf{P}\{\widehat{\tau} \in d y / A(a, x)\} \mathbf{P}\left\{\beta\left(\tau^{-}(a, 1)\right) \notin B / \xi(0)=1\right\} \\
& =1-\mathbf{P}\{\widehat{\tau}<\infty / A(a, x)\} \mathbf{P}\left\{\beta\left(\tau^{-}(a, 1)\right) \notin B / \xi(0)=1\right\} \\
& \leq 1-\delta\left(1-\frac{\delta}{1+\delta}\right)=1-\frac{\delta}{1+\delta}=1-\varepsilon .
\end{aligned}
$$

Now, we want to prove that Item 2) takes place as well. Here we need some auxiliary results.

\section{Auxiliary Results}

Let us consider a system $G I / G I / 1 /\left(Q_{1}, Q_{2}\right)$. Let $\xi(t)$ denote the number of customers in this system at time $t$ including customers in service, if any.

We put

$\tau=\inf \{t: \xi(t)=0\}-$ the first busy period of the system;

$\delta=\inf \{t>\tau: \xi(t) \neq 0\}-\tau$ - the first idle time.

Set

$$
q_{i}(s)=\int_{0}^{\infty} e^{-s x} d Q_{i}(x), \quad m_{i}=\int_{0}^{\infty} x d Q_{i}(x) .
$$

Throughout this section we shall assume that at least one of the distributions $Q_{1}(x)$, $Q_{2}(x)$ is non-lattice, $\int_{0}^{\infty} x^{2} d Q_{i}(x)<\infty, \mathrm{i}=1,2$ and $m_{2}<m_{1}$, i.e., the traffic intensity of the system $\rho=m_{2} / m_{1}$ is less than 1 .

Let $\lambda \geq 0$ be fixed. We have

$$
1-q_{1}(s) q_{2}(\lambda-s)=1-q_{2}(\lambda) \int_{-\infty}^{\infty} e^{-s x} d Q(x, \lambda),
$$


where $Q(x, \lambda)$ is some distribution function such that $\int_{-\infty}^{\infty} x^{2} d Q(x, \lambda)<\infty$ for all $\lambda \geq 0$.

Let $\xi_{i}(\lambda), i \geq 1$ be a sequence of i.i.d. random variables having the distribution function $Q(x, \lambda)$ and $S_{n}(\lambda), n \geq 0$ be the random walk associated with $\xi_{i}(\lambda)$, i.e.,

$$
S_{n}(\lambda)=\sum_{i=1}^{n} \xi_{i}(\lambda), \quad S_{0}(\lambda)=0 .
$$

It is clear that $\mathbf{E} \xi_{i}^{2}(\lambda)<\infty, \lambda \geq 0$.

Set

$$
\begin{aligned}
& \tau_{+}(\lambda)=\min \left\{n>0: S_{n}(\lambda)>0\right\}, \quad \tau_{-}(\lambda)=\min \left\{n>0: S_{n}(\lambda) \leq 0\right\} \\
& \eta_{ \pm}(\lambda)=S_{\tau_{ \pm}(\lambda)}(\lambda) .
\end{aligned}
$$

From (4.1) we have (see, for example, [1]).

Lemma 4.1: For any $\lambda>0,|z| \leq 1$ the following factorisation

$$
1-q_{1}(s) q_{2}(\lambda-s)=q_{+}(s, \lambda) q_{-}(s, \lambda), \quad 0 \leq \operatorname{Re} s \leq \lambda
$$

holds true. Here the functions $q_{+}(s, \lambda), q_{-}(s, \lambda)$ are regular and bounded in the halfplains $\operatorname{Re} s>0$, Re $s<\lambda$, respectively, don't vanish there and $q_{+}(\infty, \lambda)=1$. The decomposition (4.2) is unique.

Moreover,

$$
q_{ \pm}(s, \lambda)=1-\mathbf{E}\left\{\left(q_{2}(\lambda)\right)^{\tau_{ \pm}(\lambda)} e^{-s \eta_{ \pm}(\lambda)}\right\}, \quad \pm \operatorname{Re} s \geq 0
$$

If $\int_{-\infty}^{\infty} \exp (-a x)|f(x)| d x<\infty$ we define the projectors $I_{ \pm}$as follows

$$
I_{+}\left[\int_{-\infty}^{\infty} e^{-s x} f(x) d x\right]=\int_{0}^{\infty} e^{-s x} f(x) d x, \quad I_{-}=I-I_{+}, \quad \operatorname{Re} s=a,
$$

where $I$ stands for the identity operator.

Theorem 4.1: [2] For $0<s \leq u<\lambda, n \geq 1$ we have

$$
\int_{0}^{\infty} e^{-(s-\lambda) x} \mathbf{E}\left\{e^{-\lambda \tau-u \delta} / D(n+1, x)\right\} d x=I_{+}\left[\frac{q_{2}^{n-1}(\lambda-s)\left(q_{+}(s, \lambda)-q_{+}(u, \lambda)\right)}{(s-u) q_{+}(s, \lambda)}\right] .
$$

Let

$$
\begin{aligned}
& D_{+}(y, \lambda)=\mathbf{E}\left\{\left(q_{2}(\lambda)\right)^{\tau_{+}(\lambda)} ; \eta_{+}(\lambda)<y\right\}, \quad D_{+}(y)=D_{+}(y, 0)=\mathbf{P}\left(\eta_{+}(0)<y\right), \\
& D_{+}^{n *}(x, \lambda)=\int_{0}^{x} D_{+}^{(n-1) *}(x-y, \lambda) d D_{+}(y, \lambda), \quad n \geq 2 ; \quad D_{+}^{1 *}(\cdot, \cdot)=D_{+}(\cdot, \cdot) .
\end{aligned}
$$

and

$$
H_{+}(x, \lambda)=\sum_{k=0}^{\infty} D_{+}^{k *}(x, \lambda)
$$


Using (4.3) and the next obvious identity

$$
q_{2}^{n-1}(\lambda-s)=\int_{0}^{\infty} e^{-(\lambda-s) x} d Q_{2}^{(n-1) *}(x),
$$

we can convert identity (4.4) which gives

Theorem 4.2:

$$
e^{\lambda x} \mathbf{E}\left\{e^{-\lambda \tau-u \delta} / D(n+1, x)\right\}=\int_{0}^{\infty} Q_{+}(x+y, \lambda, u) e^{-\lambda y} d F_{2}^{(n-1) *}(y) .
$$

where

$$
Q_{+}(x, \lambda, u)=\int_{0}^{x} e^{u(x-y)} \int_{x-y}^{\infty} e^{-u z} d D_{+}(z, \lambda) d H_{+}(y, \lambda)
$$

and

$$
\mathbf{P}\{\delta \geq v / D(n+1, x)\}=\int_{0}^{\infty} \int_{0}^{x+y} \mathbf{P}\left\{\eta_{+}(0) \geq v+x+y-t\right\} d H_{+}(t) d F_{2}^{(n-1) *}(y),
$$

where $H(u)$ is a renewal function generated by the distribution $\mathbf{P}\left\{\eta_{+}(0)<u\right\}$.

Since

$$
\left.\frac{d}{d s} q_{1}(s) q_{2}(-s)\right|_{s=0}=m_{2}-m_{1}<0
$$

we can find $\alpha>0, \varepsilon>0$ such that

$$
\mathbf{E} \xi_{1}(\lambda)=-\left.\frac{d}{d s} q_{1}(s) q_{2}(\lambda-s)\right|_{s=0} \geq \alpha, \quad 0 \leq \lambda \leq \varepsilon,
$$

and until the end of this section we assume that (4.6) holds. Due to (4.6) we can assert that

$$
\mathbf{E} \eta_{+}^{2}(\lambda)<\infty, \quad 0 \leq \lambda \leq \varepsilon .
$$

Lemma 4.3: For every $n \geq 1$ we have

$$
\sup _{x \geq 0} \mathbf{E}\{\delta / D(n+1, x\}<\infty .
$$

Proof: From (4.5) it follows

$$
\mathbf{E}\{\delta / D(n+1, x)\}=\int_{0}^{\infty} \int_{0}^{x+y} \int_{x+y-t}^{\infty} \mathbf{P}\left\{\eta_{+}(0) \geq v\right\} d v d H_{+}(t) d F_{2}^{(n-1) *}(y) .
$$

For the function

$$
\widehat{Q}(z)=\int_{0}^{z} \int_{z-t}^{\infty} \mathbf{P}\left\{\eta_{+}(0) \geq v\right\} d v d H_{+}(t)
$$


due to the key renewal theorem we have

$$
\lim _{z \rightarrow \infty} \widehat{Q}(z)=\frac{1}{\mathbf{E} \eta_{+}(0)} \int_{0}^{\infty} x \mathbf{P}\left\{\eta_{+}(0) \geq x\right\} d x=\frac{\mathbf{E} \eta_{+}^{2}(0)}{2 \mathbf{E} \eta_{+}(0)}<\infty
$$

which, obviously, implies (4.8).

Remark 4.1: In what follows the absolute constants (which may be different in the different correlations) will be denoted by $C_{i}$.

Lemma 4.4.: For any $n$ there exist $C_{1}, C_{2}, C_{3}<\infty$ such that

$$
\mathbf{E}\{\tau / D(n+1, x)\}<C_{1} n+C_{2} x+C_{3} .
$$

Proof: Let us introduce some notations to be used below.

If $\varkappa$ is a departure epoch, then a remaining arrival time at the moment $\varkappa$ is denoted by $\zeta(\varkappa)$.

Since the traffic intensity of the system is less than 1 , we have

$$
\sup _{x \geq 0} \mathbf{E}\{\tau / A(1, x)\}=C<\infty
$$

Put $\bar{\tau}=\inf \{t>0: \xi(t)=n-1\}$. Obviously, $\mathbf{E}\{\bar{\tau} / A(n, x)\}=\mathbf{E}\{\tau / A(1, x)\}$. We have

$$
\begin{aligned}
& \mathbf{E}\{\tau / A(n, x)\}=\mathbf{E}\{\bar{\tau} / A(n-1, x)\} \\
&+\int_{0}^{\infty} \mathbf{E}\{\tau / A(n-1, y)\} d \mathbf{P}\{\zeta(\bar{\tau})<y / A(n, x)\} \\
& \leq C+\int_{0}^{\infty} \mathbf{E}\{\tau / A(n-1, y)\} d \mathbf{P}\{\zeta(\bar{\tau})<y / A(n, x)\} .
\end{aligned}
$$

From (4.9) and (4.10) we have $\sup _{x \geq 0} \mathbf{E}\{\tau / A(2, x)\} \leq 2 C$. This and (4.10) yields

$$
\sup _{x \geq 0} \mathbf{E}\{\tau / A(n, x)\} \leq C n .
$$

Let $N(x)$ denote the number of events in the renewal process generated by distribution $Q_{1}(x)$ on interval $[0, x]$. It is well-known that $\mathbf{E} N(x) \leq C_{4}(x+1), x \geq 0$. We have

$$
\begin{aligned}
\mathbf{E}\{\tau / D(n, x)\} & =x+\sum_{m=0}^{\infty} \int_{0}^{\infty} \mathbf{E}\{\tau / A(n+m, y)\} \mathbf{P}\left\{\beta^{-}(x) \in d y, N(x)=m\right\} \\
& \leq x+C \sum_{m=0}^{\infty} \int_{0}^{\infty}(n+m) \mathbf{P}\left\{\beta^{-}(x) \in d y, N(x)=m\right\} \\
& =x+C n+C \sum_{m=0}^{\infty} m \mathbf{P}\{N(x)=m\} \\
& =x+C n+C \mathbf{E} N(x) \leq C n+x\left(1+C C_{4}\right)+C C_{4} .
\end{aligned}
$$




\section{Proof of Theorem 2}

Below we use the fact that $\mathbf{P}\left\{\tau^{-}(a, 1)<x, \beta\left(\tau^{-}(a, 1)\right)<y / D(b, u)\right\}=\mathbf{P}\{\tau<x, \delta<$ $y / D(b-a, u)\}$, where $\tau$ and $\delta$ are the first busy period and the first idle time, respectively, of the system $G I / G I / 1\left(G_{2}, F_{2}\right)$ providing that it starts to work at the moment that $b-a$ customers are in the queue and the first departure epoch occurs in time $u$.

Lemma 5.1: Let $\pi_{+}\{\cdot\}$ denote the invariant probabilistic measure of the kernel $Q(x, d u)$, then

$$
\int_{0}^{\infty} x \pi_{+}(d x)<\infty .
$$

Proof: We have

$$
\begin{aligned}
& \left.\int_{0}^{\infty} x \pi_{+}\{d x\}\right)=\int_{0}^{\infty} x \int_{0}^{\infty} Q(y, d x) \pi_{+}(d y) \\
& =\int_{0}^{\infty} x \int_{0}^{\infty} \int_{0}^{\infty} \mathbf{P}\left\{\beta\left(\tau^{+}(b, 1)\right) \in d u / A(a, y)\right\} \mathbf{P}\left\{\beta\left(\tau^{-}(a, 1)\right) \in d x / D(b, u)\right\} \pi_{+}(d y) \\
& \leq \sup _{u \geq 0} \int_{0}^{\infty} x \mathbf{P}\left\{\beta\left(\tau^{-}(a, 1)\right) \in d x / D(b, u)\right\} \\
& =\sup _{u \geq 0} \int_{0}^{\infty} x \mathbf{P}\{\delta \in d x / D(b-a, u)\}<\infty .
\end{aligned}
$$

Here in the last line we use Lemma 4.

Now we can prove Item 2) of Theorem 3 . We have

$$
\begin{aligned}
& \int_{0}^{\infty} \pi_{+}\{d x\} \int_{0}^{\infty} \mathbf{P}\left\{\tau^{-}(a, 1)>u / A(a, x)\right\} d u=\int_{0}^{\infty} \pi_{+}\{d x\} \mathbf{E}\left\{\tau^{-}(a, 1) / A(a, x)\right\} \\
& \leq \int_{0}^{\infty} \pi_{+}\{d x\}\left(x+\mathbf{E}\left\{\tau^{-}(a, 1) / \xi(0)=1\right\}\right) \\
& =\int_{0}^{\infty} x \pi_{+}\{d x\}+\mathbf{E}\left\{\tau^{-}(a, 1) / \xi(0)=1\right\}
\end{aligned}
$$

and

$$
\begin{aligned}
& \mathbf{E}\left\{\tau^{-}(a, 1) / \xi(0)=1\right\}=\mathbf{E}\left\{\tau^{+}(b, 1) / \xi(0)=1\right\} \\
& +\int_{0}^{\infty} \mathbf{E}\left\{\tau^{-}(a, 1) / D(b, w)\right\} \mathbf{P}\left\{\beta\left(\tau^{+}(b, 1)\right) \in d w / \xi(0)=1\right\} \\
& \leq \mathbf{E}\left\{\tau^{+}(b, 1) / \xi(0)=1\right\}+\int_{0}^{\infty}\left(C(b-a)+C_{1} w\right) \mathbf{P}\left\{\beta\left(\tau^{+}(b, 1)\right) \in d w / \xi(0)=1\right\} \\
& =C+\mathbf{E}\left\{\tau^{+}(b, 1) / \xi(0)=1\right\}+C_{1} \mathbf{E}\left\{\beta\left(\tau^{+}(b, 1)\right) / \xi(0)=1\right\}<\infty .
\end{aligned}
$$


Here the last inequality follows from the fact that $\tau^{+}(b, 1)$ and $\beta\left(\tau^{+}(b, 1)\right)$ are, in fact, the first moment that the queue length of a system $G I / G I / 1\left(G_{1}, F_{1}\right)$ reaches the fixed level $b$ and the remaining service time at that moment, respectively. Now Item 2) of Theorem 3 follows from (5.1) and Lemma 4.

Now from (3.1) we have

$$
\lim _{t \rightarrow \infty} \mathbf{P}\{\xi(t)=l / A(a, x)\}=\frac{1}{m} \int_{0}^{\infty} \pi_{+}(d y) \int_{0}^{\infty} U_{l}(t, y) d t=P_{l},
$$

where

$$
m=\int_{0}^{\infty} \pi_{+}\{d x\} \mathbf{E}\left\{\tau^{-}(a, 1) / A(a, x)\right\}
$$

\section{Application to the System G/M-M/1}

Let $F_{1}(x)=1-e^{-\nu x}, F_{2}(x)=1-e^{-\mu x}$, and $G_{1}(x)=G_{2}(x)=G(x)$. In view of Condition A we have $\int_{0}^{\infty} x d G(x)>1 / \mu$.

For $n \geq 2$ let us introduce the following random times

$$
\begin{aligned}
\tau^{+}(b, n) & =\inf \left\{t>\tau^{-}(a, n-1): \xi(t)=b\right\} \\
\tau^{-}(a, n) & =\inf \left\{t>\tau^{+}(b, n): \xi(t)=a\right\} .
\end{aligned}
$$

If $\xi(0)=b$, then, due to the memoryless property of the exponential distribution, the epochs $\tau^{+}(b, n), n \geq 0, \tau^{+}(b, 0)=0$ are Markovian for process $\xi(t)$ and the random variables $T_{i}(b)=\tau^{+}(b, i+1)-\tau^{+}(b, i), i \geq 0$ have the same distribution.

For simplicity of notation, we shall use the following abbreviations: $\tau^{+}(b), \tau^{-}(a)$, $\beta(a), \mathbf{P}_{k}\{\cdot\}, \mathbf{E}_{k}\{\cdot\}$ instead of $\tau^{+}(b, 1), \tau^{-}(a, 1), \beta\left(\tau^{-}(a, 1)\right), \mathbf{P}\{\cdot / \xi(0)=k\}, \mathbf{E}\{\cdot / \xi(0)=$ $k\}$, respectively.

The key renewal theorem with respect to the epochs $\tau^{+}(b, i)$ gives

$$
\left.P_{l}=\lim _{t \rightarrow \infty} \mathbf{P}_{b}\{\xi(t)=l\}=\frac{1}{m} \int_{0}^{\infty} \mathbf{P}_{b}\left\{\xi(t)=l, T_{0}(b)>t\right)\right\} d t,
$$

where $m=\mathbf{E}_{b}\left\{T_{0}(b)\right\}$.

Let

$$
\begin{aligned}
W_{l}(b, a) & =\int_{0}^{\infty} \mathbf{P}_{b}\left\{\xi(t)=l, \tau^{-}(a)>t\right\} d t, \\
V_{l}(k, b) & =\int_{0}^{\infty} \mathbf{P}_{k}\left\{\xi(t)=l, \tau^{+}(b)>t\right\} d t, \quad k<b .
\end{aligned}
$$

We have

$$
\int_{0}^{\infty} \mathbf{P}_{b}\left(\xi(t)=l, T_{0}(b)>t\right) d t=W_{l}(b, a)+\int_{0}^{\infty} \mathbf{P}_{b}\left(\xi(t)=l, \tau^{-} \leq t, T_{0}(b)>t\right) d t .
$$


Let $N(x)$ denote the number of customers departured in the time interval of length $x$.

We have

$$
\begin{aligned}
& \int_{0}^{\infty} \mathbf{P}_{b}\left\{\xi(t)=l, \tau^{-}(a) \leq t, T_{0}(b)>t\right\} d t= \\
& =\sum_{k=0}^{\infty} \int_{0}^{\infty} d t \int_{0}^{t} \int_{0}^{t-u} \mathbf{P}_{b}\left\{\xi(t)=l, T_{0}(b)>t / \tau^{-}(a)=u, \beta(a)=z, N(z)=k\right\} \\
& \quad \times \frac{e^{-\nu z}(\nu z)^{k}}{k !} \mathbf{P}_{b}\left\{\tau^{-}(b) \in d u, \beta(a) \in d z\right\} \\
& +\int_{0}^{\infty} \int_{0}^{t} \int_{t-u}^{\infty} \mathbf{P}_{b}\left\{\xi(t)=l, T_{0}(b)>t / \tau^{-}(a)=u, \beta(a)=z\right\} \\
& =\sum_{k=0}^{a-1} V_{l}(a-k+1, b) \int_{0}^{\infty} \frac{e^{-\nu z}(\nu z)^{k}}{k !} \mathbf{P}_{b}\{\beta(a) \in d z\} \\
& +V_{l}(1, b) \sum_{k=a}^{\infty} \int_{0}^{\infty} \frac{e^{-\nu z}(\nu z)^{k}}{k !} \mathbf{P}_{b}\{\beta(a) \in d z\}+\int_{0}^{\infty} \mathbf{P}_{b}\{\beta(a) \in d z\} \int_{0}^{z} d_{a, l}(t) d t \\
& \left.=V_{l}(1, b)+\sum_{k=0}^{a-1}\left(V_{l}(a-k+1, b)-V_{l}(1, b)\right) Q_{k}(\nu) \in d z\right\} d t= \\
& \quad+\int_{0}^{\infty} \mathbf{P}_{b}\{\beta(a) \in d z\} \int_{0}^{z} d_{a, l}(t) d t
\end{aligned}
$$

where

$$
\begin{aligned}
& Q_{k}(\nu)=\int_{0}^{\infty} \frac{e^{-\nu z}(\nu z)^{k}}{k !} \mathbf{P}_{b}\{\beta(a) \in d z\} \\
& d_{n, l}(t)=\left\{\begin{array}{ccc}
I(n \geq l) \frac{e^{-\nu t}(\nu t)^{n-l}}{(n-l) !} & \text { if } \quad l>0, \\
\sum_{k=n}^{\infty} \frac{e^{-\nu t}(\nu t)^{k}}{k !} & \text { if } \quad l=0 .
\end{array}\right.
\end{aligned}
$$

So we have

Theorem 6.1: For the ergodic distribution of the number of customers in the system $\mathrm{G} / \mathrm{M}-\mathrm{M} / 1$ the following representation holds true

$$
\begin{aligned}
P_{l}=\frac{1}{m} & {\left[W_{l}(b, a)+V_{l}(1, b)+\sum_{k=0}^{a-1}\left(V_{l}(a-k+1, b)-V_{l}(1, b)\right) Q_{k}(\nu)\right.} \\
& \left.+\int_{0}^{\infty} \mathbf{P}_{b}\{\beta(a) \in d z\} \int_{0}^{z} d_{a, l}(t) d t\right] .
\end{aligned}
$$


It remains to find representations for $V_{l}(k, b), W_{l}(b, a)$ and for distribution $\beta_{a}(1)$. To this end, we use the potential method proposed by Koroluyk in [7].

We present in the following detailed arguments only for the functional $V_{l}(k, b)$. For the other two functionals we formulate the final representations.

For the function $\Phi_{n}(t)=\mathbf{P}_{n}\left(\xi(t)=l, \tau^{+}(b)>t\right)$ the standard arguments give

$$
\begin{aligned}
\Phi_{n}(t) & =\sum_{k=0}^{n-1} \int_{0}^{t} \Phi_{n-k+1}(t-u) \frac{e^{-\nu u}(\nu u)^{k}}{k !} d G(u)+ \\
& +\sum_{k=n}^{\infty} \int_{0}^{t} \Phi_{1}(t-u) \frac{e^{-\nu u}(\nu u)^{k}}{k !} d G(u)+d_{n, l}(t) \cdot(1-G(t))
\end{aligned}
$$

for $0<n<b$ and $\Phi_{b}(t)=0$.

Put

$$
\begin{aligned}
\phi_{n} & =\int_{0}^{\infty} \Phi_{n}(t) d t\left(=V_{l}(n, b)\right), \quad p_{k}=\frac{1}{(k+1) !} \int_{0}^{\infty} e^{-\nu t}(\nu t)^{k+1} d G(t), \quad k \geq-1, \\
q_{n, l} & =\int_{0}^{\infty} d_{n, l}(t)(1-G(t)) d t= \\
& =\left\{\begin{array}{c}
\frac{I(n \geq l)}{(n-1) !} \int_{0}^{\infty} e^{-\nu t}(\nu t)^{n-l}(1-G(t)) d t, \\
\int_{0}^{\infty} x d G(x)-\frac{1}{k !} \sum_{k=0}^{n-1} \int_{0}^{\infty} e^{-\nu t}(\nu t)^{k}(1-G(t)) d t, \quad \text { if } \quad l=0 .
\end{array}\right.
\end{aligned}
$$

then (6.3) leads to the following boundary problem

$$
\phi_{n}-\sum_{k=-1}^{n-2} \phi_{n-k} p_{k}-\phi_{1} \sum_{k=n-1}^{\infty} p_{k}=q_{n, l}, \quad 0<n<b
$$

with the boundary condition

$$
\phi_{b}=0 .
$$

To solve the problem (6.4), (6.5) we use Korolyuk's approach [7]. To this end we introduce some necessary notions.

Note that $\sum_{k=-1}^{\infty} p_{k}=1$, and hence the numbers $p_{k}, k \geq-1$ can be treated as distributions of jumps of a semi-continuous (from below) random walk. We can easily find that

$$
\sum_{k=-1}^{\infty} z^{k} p_{k}=g(\nu(1-z)) / z, \quad 0<|z| \leq 1
$$

where $g(s)=\int_{0}^{\infty} \exp (-s x) d G(x)$. Let the sequence $R_{n}, n \geq 0$ be a potential of that walk, i.e., this sequence is defined to be

$$
\sum_{n=1}^{\infty} z^{n} R_{n}=\frac{z}{g(\nu(1-z))-z}, \quad|z|<u_{\nu}
$$


where $u_{\nu}$ is the smallest solution of the equation $g(\nu(1-z))=z$ on the interval $[0,1]$.

Let us return to the problem (6.4), (6.5) and rewrite (6.4) in the form

$$
\sum_{k=-1}^{n-1} p_{k} \phi_{n-k}-\phi_{n}=\psi_{n, l}, \quad 0<n<b
$$

where

$$
\psi_{n, l}=-q_{n, l}-\phi_{1} r_{n}, \quad r_{n}=\sum_{k=n}^{\infty} p_{k} .
$$

According to Korolyuk [7] we have

$$
\phi_{n}=C R_{n}+\sum_{k=1}^{n-1} R_{n-k} \psi_{k, l}
$$

Putting in (6.8) $n=1$ gives $C=\phi_{1} / R_{1}=g(\nu) \phi_{1}$. The boundary condition $\phi_{b}=0$ gives

$$
\phi_{1}=\frac{\sum_{k=1}^{b} R_{b-k} q_{k, l}}{g(\nu) R_{b}-\sum_{k=1}^{b-1} R_{b-k} r_{k}} .
$$

Finally, we have

Theorem 6.2: For $0<n<b$

$$
V_{l}(n, b)=\phi_{1}\left(g(\nu) R_{n}-\sum_{k=1}^{n-1} R_{n-k} r_{k}\right)-\sum_{k=1}^{n-1} R_{n-k} q_{k, l} .
$$

Now we state the representations for $W_{l}(b, a), \mathbf{P}_{b}\{\beta(a)>z\}$.

Theorem 6.3:

$$
\begin{aligned}
& W_{l}(b, a)=u_{\mu}^{l-1} \mu^{-1} R_{b-a-1}-\sum_{k=1}^{b-a} R_{b-a-k} q_{k-l}(0), \\
& \mathbf{P}_{b}\{\beta(a)>z\}=\mu\left(R_{b-a-1}+\delta_{b-a, 1}\right) \sum_{k=0}^{\infty} u_{\mu}^{k} q_{k}(z)-\mu \sum_{k=1}^{b-a} R_{b-a-k} q_{k-1}(z),
\end{aligned}
$$

where

$$
q_{k}(z)=\frac{1}{k !} \int_{0}^{\infty} e^{-\mu v}(\mu v)^{k}(1-G(v+z)) d v \quad z \geq 0 .
$$

and $\delta_{i, j}$ is the Kronecker's symbol.

Remark 6.1: In formulas (6.11), (6.12) the sequence $R_{k}, k=1,2, \ldots$ is defined by (6.6) with $\mu$ instead of $\nu$. 


\section{Examples}

Let $G(x)$ be a Paretto distribution with parameters $x_{0}=2$ i $\alpha=5$, i.e. $G(t)=$ $1-16 / t^{4}, t \geq 2$ and take $a=5, \quad b=10, \quad \nu=0.5, \quad \mu=2$.

To calculate the function $R_{k}$ we use the following recurrent formula:

$$
R_{k+1}=R_{1}\left(R_{k}-\sum_{n=0}^{k-1} p_{n} R_{k-n}\right), \quad k \geq 1,
$$

where $R_{1}=(g(\nu))^{-1}$ if we use the representation (6.10) and $R_{1}=(g(\mu))^{-1}$ if we use the representations (6.11), (6.12).

Table 1 and Figure 1 show results for the probabilities $P_{l}$ obtained by applying formulas (6.2) and (6.9)-(6.12).

Table 2 and Figure 2 show results for the probabilities $P_{l}$ obtained for the system with different parameter $\nu=0.25$. (The other parameters are the same)

\begin{tabular}{|c|c|c|c|}
\hline$l$ & $P_{l}$ & $l$ & $P_{l}$ \\
\hline 0 & 0.25280 & 7 & 0.01067 \\
\hline 1 & 0.31929 & 8 & 0.00511 \\
\hline 2 & 0.18487 & 9 & 0.00191 \\
\hline 3 & 0.10705 & 10 & 0.00018 \\
\hline 4 & 0.06199 & 11 & $1.5850 \mathrm{e}-6$ \\
\hline 5 & 0.03589 & 12 & $1.4229 \mathrm{e}-8$ \\
\hline 6 & 0.02023 & & \\
\hline
\end{tabular}

Table 1: Ergodic distribution for $\nu=0.5$ 


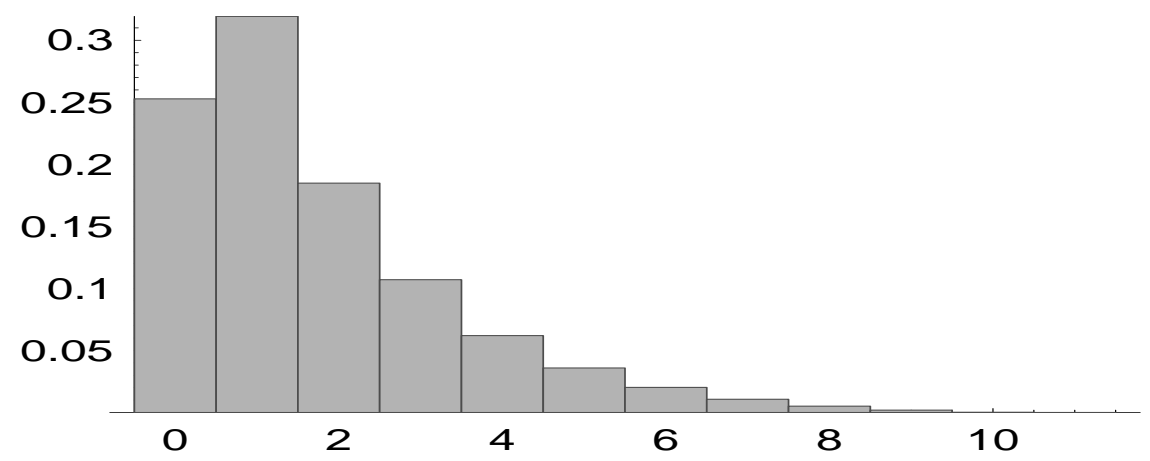

Figure 1: Ergodic distribution for $\nu=0.5$

\begin{tabular}{|c|c|c|c|}
\hline$l$ & $P_{l}$ & $l$ & $P_{l}$ \\
\hline 0 & 0.00395 & 7 & 0.19464 \\
\hline 1 & 0.01063 & 8 & 0.16543 \\
\hline 2 & 0.02058 & 9 & 0.10923 \\
\hline 3 & 0.04015 & 10 & 0.01360 \\
\hline 4 & 0.07901 & 11 & 0.00012 \\
\hline 5 & 0.15617 & 12 & $1.0962 \mathrm{e}-6$ \\
\hline 6 & 0.20649 & & \\
\hline
\end{tabular}

Table 2: Ergodic distribution for $\nu=0.25$

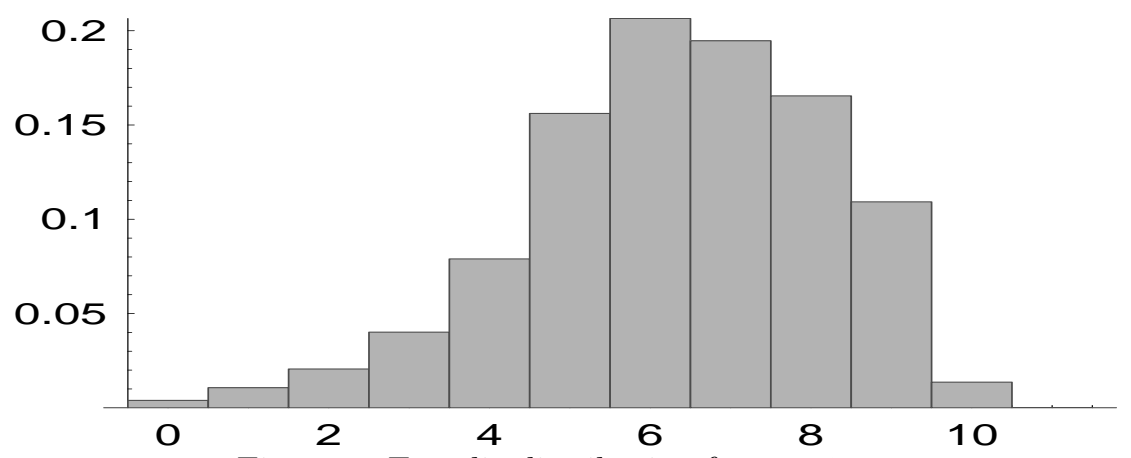

Figure 2: Ergodic distribution for $\nu=0.25$ 


\section{References}

[1] Borovkov, A.A., Probabilistic Processes in Queueing Theory, M. Nauka 1972.

[2] Bratiychuk, M.S., Limit theorems for some characteristics of system $G I / G I / 1$, Exploring Stochastic Laws (1995), 77-90.

[3] Choi, D., Knessl, C. and Tier, C., A queueing system with queue length dependent service times with applications to cell discarding in ATM networks, J. of Appl. Math. and Stoch. Anal. 12:1 (1999), 35-62.

[4] Dshalalow, J.H., Queueing systems with state-dependent parameters, In: Frontiers in Queueing, CRC Press, Boca Raton, FL 1997.

[5] Gong, W.B., Yan, A. and Cassandras, C.G., The M/G/1 queue with queue-length dependent arrival rate, Stoch. Models 8 (1992), 733-741.

[6] Kemperman, J.H.B., The oscillating random walk. Stoch. Proc. Appl. 2:1 (1974), 1-29.

[7] Korolyuk, V.S., Boundary Problems for Compound Poisson Processes, Naukowa Dumka, Kiev 1975.

[8] Li, S.Q., Overload control in finite message storage buffer, IEEE Trans. Commun. 37 (1989), 703-712.

[9] Lotov, V.I., On oscillating random walks, Siber. Math. J. 37:4 (1996).

[10] Shurenkov, V.M., On the Markov renewal theory, Theory Probab. and its Appl. 29:2 (1984), 248-263.

[11] Sriaram, K., McKinney, R.S., and Sherif, M.H., Voice packetization and compression in broadband ATM networks, IEEE J. on Selected Areas in Commun. 9:3 (1991), 294-304.

[12] Takagai, H., Analysis of a finite capacity M/G/1 queue with resume level, Perf. Eval. 5 (1985), 197-203. 


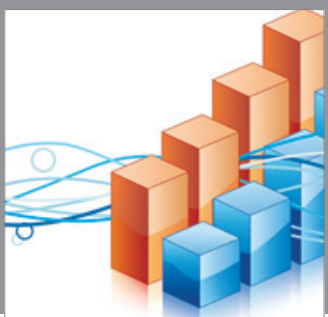

Advances in

Operations Research

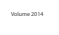

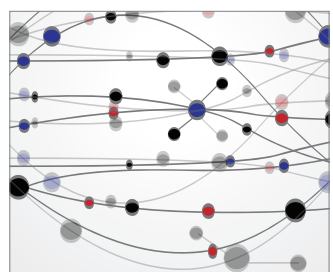

\section{The Scientific} World Journal
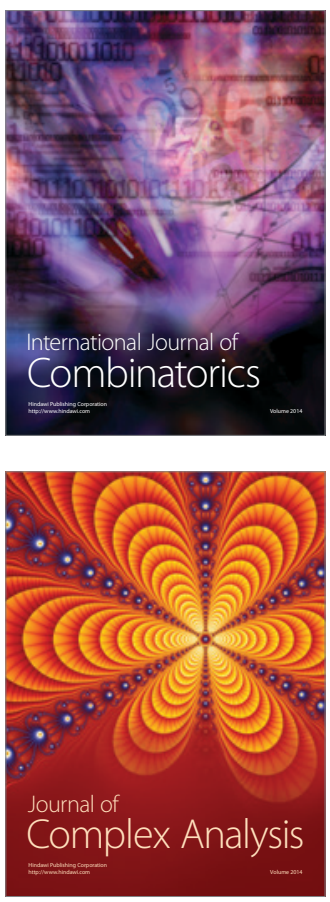

International Journal of

Mathematics and

Mathematical

Sciences
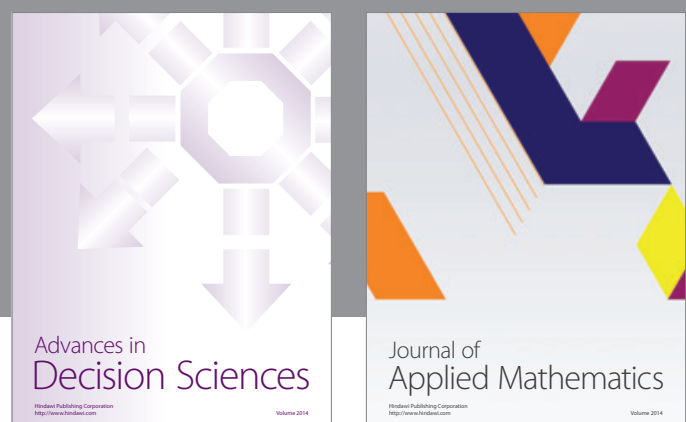

Journal of

Applied Mathematics
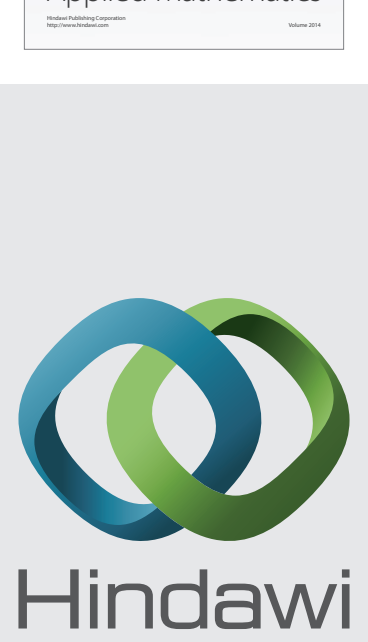

Submit your manuscripts at http://www.hindawi.com
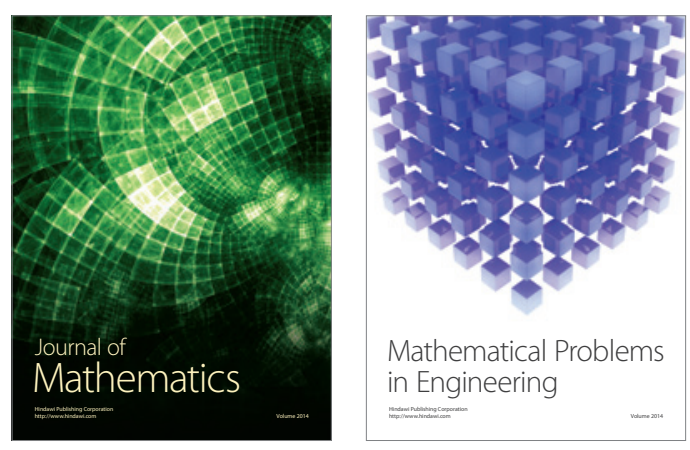

Mathematical Problems in Engineering
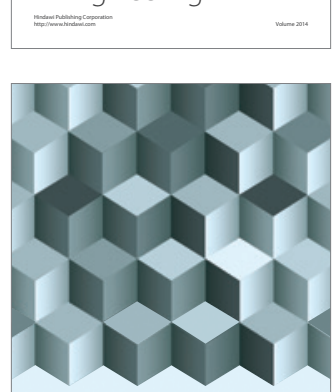

Journal of

Function Spaces
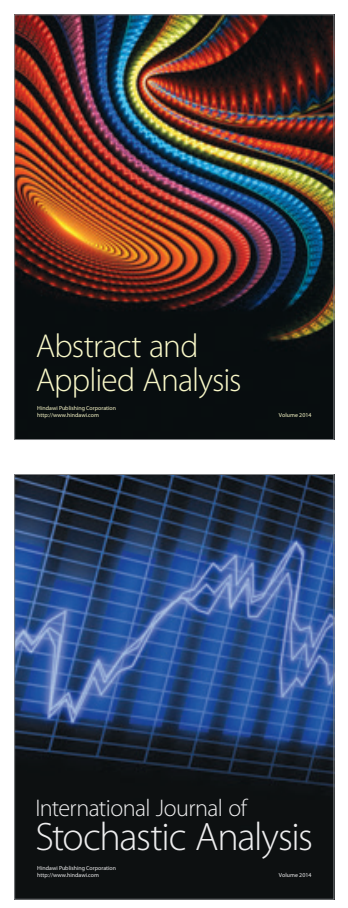

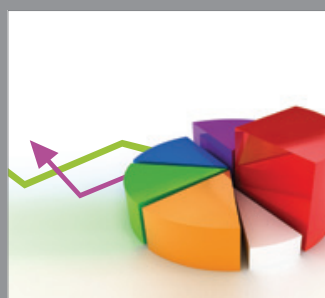

ournal of

Probability and Statistics

Promensencen
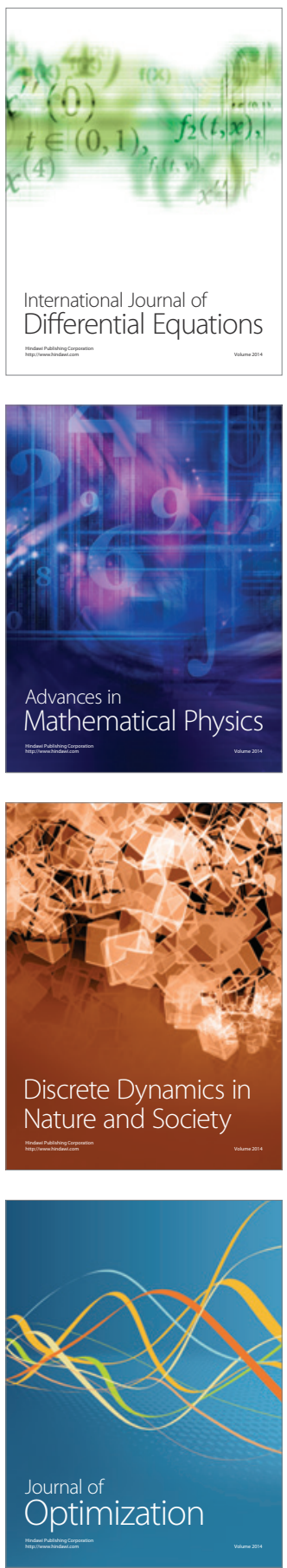